\title{
Análise desenvolvimentista e do desempenho do salto vertical em escolares
}

\section{Developmental assessment and performance analysis of vertical jump in schoolchildren}

Pedro Paulo Deprá ${ }^{1}$

Dalker Roberto Walter ${ }^{2}$

Resumo - O salto vertical além de possuir complexidade coordenativa, agrega ao indivíduo diferentes experiências motoras. O objetivo deste trabalho foi verificar quais movimentos da sequência desenvolvimentista apresentados por escolares do ensino fundamental têm associação com a faixa etária e o desempenho no salto vertical. Fizeram parte deste estudo 137 escolares de um colégio do município de Maringá, Paraná, com idade entre 7-10 (anos), estatura entre 1,19 e 1,63 metros, massa entre 20 e $60 \mathrm{~kg}$, de ambos os sexos e matriculados até a quarta série do ensino fundamental. Como instrumentos da pesquisa, foram utilizados a matriz de Gallahue e Ozmun e uma plataforma de salto. No procedimento experimental, cada criança realizou três saltos. Em crianças de sete anos, constatou-se a presença de elevação de braços coordenados e simultânea. Em crianças de nove anos, constatou-se a presença de agachamento preparatório inconsistente e a ação de braços não coordenados com o tronco e a perna. Foi observada prevalência da ação dos membros superiores quando consideradas as associações significativas entre os elementos da sequência desenvolvimentista e o desempenho do salto vertical. Na faixa etária entre 7-10 (anos), a faixa etária e o desempenho do salto estão associados aos elementos da sequência desenvolvimentista da configuração total do corpo humano, preponderantemente relacionados aos membros superiores.

Palavras-chave: Biomecânica; Destreza motora; Desenvolvimento infantil.

Abstract - The vertical jump involves different levels of skill complexity and offers the individual a wide range of motor experiences. This study aimed to determine which movements in the developmental sequence performed by schoolchildren are associated with age and vertical jump performance. The sample consisted of 137 elementary school children of both sexes, aged 7-10y, with height of 1.19-1.63 $\mathrm{m}$, and weight of 20-60 kg. All children were selected from first- to fourth-grade classrooms of a public school of the city of Maringá, state of $\mathrm{Pa}$ raná, Brazil. A Gallahue \& Ozmun matrix and a jumping platform were used as research instruments. During the experiment, each child performed three jumps. Simultaneous and coordinated upward arm lift was observed in 7-year-old children. Nine-year-old children showed inconsistent preparatory crouch and lack of coordination between upper limb and trunk/lower limb movements. A prevalence of upper limb motor acts was observed when considering the significant associations found between elements of the developmental sequence and vertical jump performance. In children aged 7-10y, age group and jumping performance are associated with elements of the developmental sequence of total body configuration, especially with regard to the upper limbs.

Key words: Biomechanics; Child development; Motor skills.

1 Universidade Estadual de Maringá. Programa de Pós-Graduação Associado em Educação Física UEM/UEL. Londrina. PR. Brasil

2 Universidade Estadual de Maringá. Curso de Educação Física. Maringá. PR. Brasil

Recebido em 16/06/11 Revisado em 23/10/11 Aprovado em 17/02/12 


\section{INTRODUÇÃO}

Durante a vida, o ser humano apresenta alterações progressivas do comportamento motor ${ }^{1}$. De forma sequencial, considerando o Modelo de Desenvolvimento Motor proposto por Gallahue, as fases motoras reflexivas, rudimentares, fundamentais e especializadas vão acontecendo ao longo da vida da criança influenciadas por fatores individuais e ambientais. Em cada uma dessas fases, a criança pode apresentar padrões de movimentos com níveis de aquisição diferenciados que dependerão das exigências mecânicas e físicas da tarefa a ser cumprida, das oportunidades de prática e de processos de encorajamentos ${ }^{1}$.

O salto vertical é uma habilidade motora de grande importância para o desenvolvimento do ser humano. Além da sua complexidade coordenativa, com o envolvimento de vários segmentos corporais ${ }^{1}$, agrega ao indivíduo diferentes experiências motoras. Essas experiências podem contribuir para o desempenho esportivo, ainda mais quando a técnica exigida depende da habilidade do salto.

As execuções incorretas desta habilidade podem trazer complicações ortopédicas ao joelho dos atletas. Na execução dos saltos, diferentes estratégias motoras adotadas pelos atletas podem levá-los a uma maior ou menor predisposição à lesão do ligamento cruzado anterior ${ }^{2}$.

Embora o salto vertical tenha sido amplamente investigado pela literatura no que se refere à causa de lesões ${ }^{3}$, custos de cirurgias e tratamentos, identificamos que em nenhum aspecto foi abordada a associação entre os movimentos da sequência desenvolvimentista, o desempenho e a faixa etária em escolares do ensino fundamental.

Os trabalhos que enfocam o salto vertical em termos do seu desempenho procuram investigar isoladamente características da idade ${ }^{4}$, da antropometria ${ }^{5}$, do gênero ${ }^{6-8}$, do nível sócioeconômico ${ }^{9}$, das variáveis mecânicas envolvidas ${ }^{10-12}$, do tipo de treinamento desenvolvido ${ }^{13}$ e do nível de desenvolvimento motor ${ }^{10}$.

Em relação ao desenvolvimento motor, uma análise da coordenação de articulações de crianças durante a realização do salto vertical foi realizada por Harrison et al. ${ }^{10}$, em relação aos estágios de desenvolvimento motor e concluíram que os padrões do quadril e do joelho foram mais efetivos para esta discriminação.

$\mathrm{Na}$ literatura, até onde sabemos, não há pesquisas que investigam a existência de associação entre os movimentos esperados na sequência desenvolvimentista do padrão motor, o desempenho do salto e a idade em crianças.

Tem-se por hipótese de que as características da sequência de movimentos da habilidade motora do salto vertical podem ser diferenciadas entre as crianças por serem influenciadas por fatores individuais.

O objetivo deste trabalho foi verificar quais movimentos da sequência desenvolvimentista, apresentados por escolares do ensino fundamental, têm associação com a faixa etária e o desempenho no salto vertical. Entende-se 
que a relevância deste estudo está no levantamento de informações que podem subsidiar a ação dos profissionais que lidam com aspectos motores de crianças.

\section{PROCEDIMENTOS METODOLÓGICOS}

Fizeram parte deste estudo 137 escolares de um colégio do município de Maringá, Paraná, com idade entre 7-10 (anos), estatura entre 1,19 e 1,63 metros, massa entre 20 e $60 \mathrm{~kg}$, de ambos os sexos e matriculados até a quarta série do ensino fundamental. Destes alunos, 10 encontravam-se com sete anos; 29 com oito anos; 54 com nove anos e 44 e com 10 anos. A divisão das crianças por faixa etária foi realizada para possibilitar um maior espectro de variáveis para análise e colaborar com futuras comparações em pesquisas desenvolvimentistas. O critério de inclusão baseou-se na assinatura do termo de consentimento dos pais e/ou responsáveis.

O estudo foi aprovado pelo Comitê Permanente de Ética em pesquisa envolvendo seres humanos - CAAE 0323-08. Todos os escolares apresentaram o Termo de Consentimento Livre e Esclarecido devidamente assinado pelos pais e/ou responsáveis.

Os saltos foram registrados durante as aulas de Educação Física. $\mathrm{Na}$ coleta de dados, foi solicitado ao aluno que executasse o salto o mais alto possível. Não houve demonstração da biomecânica do salto. A forma do salto foi autosselecionada pelas crianças. Cada criança realizou três saltos, de forma consecutiva. O tempo médio de coleta foi de cinco minutos por criança.

Como instrumentos da pesquisa, foram utilizadas a matriz de Gallahue e Ozmun ${ }^{1}$ e uma plataforma de salto.

Para identificar o estágio de desenvolvimento motor relacionado ao salto vertical das crianças, utilizou-se a matriz de Gallahue e Ozmun ${ }^{1}$. Esta matriz apresenta uma abordagem de configuração total do corpo, sendo utilizada para grupos de crianças em um ambiente informal ${ }^{14}$. Através dessa abordagem, observa-se a mecânica corporal no desempenho do salto e compara-se com padrões pré-estabelecidos de comportamentos de segmentos corporais, classificando a criança nos estágios inicial, elementar ou maduro. Cada estágio é composto por um rol de elementos motores.

A análise do desenvolvimento motor do salto vertical considerou o padrão motor de cada criança no conjunto das três execuções. Esta análise foi realizada por apenas um avaliador. Para cada salto executado, foram identificados quais elementos motores estavam presentes.

A partir deste diagnóstico, detectou-se qual estágio apresentava a maior prevalência dentre as 18 características relacionadas ao estágio motor (seis do inicial, seis do elementar e seis do maduro). O estágio que apresentava o maior número de elementos era então selecionado como representativo para o salto executado. A sétima característica (Pouso controlado bastante próximo ao ponto de partida) do padrão considerado para o estágio maduro foi um comportamento necessário para a análise do desempenho e do nível 
de desenvolvimento motor do salto vertical, válido para registro dos dados.

Para a análise das associações, foram considerados todos os elementos da sequência desenvolvimentista detectados em cada criança. Este procedimento foi tomado para que a variabilidade dos elementos da sequência desenvolvimentista pudesse ser manifestada em cada criança. Assim, foi possível determinar o estágio de desenvolvimento motor e quais elementos estavam presentes no conjunto de saltos. Os voluntários apresentaram pouca variabilidade durante a execução dos três saltos, considerando as mesmas características desenvolvimentistas.

O desempenho no salto vertical (altura de salto) foi calculado a partir do tempo de vôo (equação 1) no momento da execução. O tempo de vôo foi obtido por uma plataforma de salto construída para esta finalidade, com o objetivo de registrar o intervalo de tempo entre os momentos de saída e aterrissagem. O sistema de registro dos dados apresentou precisão de 0,001 [s], controlado por software de alta precisão utilizando a função QueryPerformanceCounter disponível na biblioteca Microsoft.

$$
\text { Altura }=\frac{1}{8} g t^{2}
$$

Equação 1 - Cálculo da altura de salto

Para a análise, foi utilizada a maior altura alcançada por cada criança nas três tentativas registradas. Na equação 1, a letra "g" representa a aceleração da gravidade e " $\mathrm{t}$ " o tempo de vôo. A confiabilidade dos saltos registrados, calculada pelo Coeficiente de Correlação Intraclasse, apresentou-se igual 0.9.

Para verificar a existência de diferenças estatisticamente significativas das variáveis antropométricas e da altura do salto entre as faixas etárias, foram utilizados os testes KrusKall-Wallis e U de Mann-Whitney adotando nível de significância de 5\%.

Para avaliar a associação entre os elementos dos estágios da sequência desenvolvimentista e o desempenho no salto vertical, foi utilizado o Teste Qui-quadrado com nível de significância estabelecido em 5\%. Não houve necessidade da utilização do Teste Exato de Fisher, pois todas as frequências esperadas foram maiores que cinco.

Para avaliar a associação entre os elementos dos estágios da sequência desenvolvimentista e a faixa etária (Tabela L x C), utilizou-se o valor do qui-quadrado para associação linear ${ }^{15}$. Sendo verificada a associação global entre as variáveis, para indicação de associações locais foi utilizado o resíduo ajustado (RA) do teste qui-quadrado ${ }^{16,17}$. Segundo Callegari-Jacques, através da análise de resíduos é possível avaliar como as diferentes caselas em tabelas L x C contribuem para o valor do qui-quadrado calculado.

$\mathrm{Na}$ avaliação do desempenho do salto vertical, o procedimento de escolha do valor de $20 \mathrm{~cm}$, para delimitar as faixas de alturas, teve como critério o valor mediano representativo para todas as faixas etárias, como pode ser observado na tabela 1. 


\section{RESULTADOS}

As crianças ( $\mathrm{n}=137$ ) foram classificadas nos três estágios de desenvolvimento motor em relação ao salto vertical. A distribuição dos estágios apresentou as seguintes frequências e percentuais: Inicial (26; 19,0\%); Elementar (107; 78,1\%); Maduro (4, 2,9\%).

Para cada sequência desenvolvimentista, foram avaliadas as associações dos elementos motores com as variáveis relacionadas à faixa etária e ao desempenho do salto. Apesar da existência de diferenças significativas entre as medidas antropométricas (estatura e massa) nas quatro faixas etárias, não foram observadas diferenças para a variável altura de salto, o que corrobora, inicialmente, a hipótese destacada na introdução sobre as diferenças baseadas nas experiências individuais das crianças e permite uma abordagem sobre as possíveis associações apontadas.

Tabela 1. Comparação dos dados antropométricos e da altura do salto vertical por faixa etária

\begin{tabular}{lccc}
\hline $\begin{array}{l}\text { Idade } \\
\text { (anos) }\end{array}$ & Estatura[m] & Massa[kg] & Altura de Salto[m] \\
\cline { 2 - 4 } & Mediana [25-75] & Mediana [25-75] & Mediana [25-75] \\
\hline 7 & $1,30[1,26-1,36]^{\mathrm{a,b}}$ & $28,50[24,25-31,00]^{\mathrm{fg,h}, \mathrm{h}}$ & $0,23[0,20-0,26]$ \\
8 & $1,35[1,28-1,38]^{\mathrm{c,d}}$ & $31,00[27,50-35,50]^{\mathrm{fi}}$ & $0,21[0,18-0,23]$ \\
9 & $1,40[1,36-1,43]^{\mathrm{a}, \mathrm{c,e}}$ & $32,00[27,75-39,25]^{\mathrm{g,j}}$ & $0,21[0,17-0,24]$ \\
10 & $1,45[1,39-1,50]^{\mathrm{b}, \mathrm{d}, \mathrm{e}}$ & $37,00[31,00-44,00]^{\mathrm{h}, \mathrm{ij}}$ & $0,22[0,18-0,26]$ \\
\hline
\end{tabular}

Nota: Diferença estatisticamente significativa entre as faixas etárias e a variável

Estatura: (a) entre 7 e 9 anos; (b) entre 7 e 10 anos; (c) entre 8 e 9 anos; (d) entre 8 e 10 anos; (e) entre 9 e 10 anos; Massa: (f) entre 7 e 8 anos; (g) entre 7 e 9 anos; (h) entre 7 e 10 anos; (i) entre 8 e 10 anos; (j) entre 9 e 10 anos.

A tabela 2 apresenta os resultados das associações entre os elementos da sequência desenvolvimentista e a faixa etária.

Tabela 2. Associação entre os elementos da sequência desenvolvimentista do salto vertical e a faixa etária das crianças.

\begin{tabular}{|c|c|c|c|c|c|c|c|c|c|c|c|c|}
\hline \multirow{3}{*}{\multicolumn{3}{|c|}{ Sequência desenvolvimentista }} & \multicolumn{8}{|c|}{ Idade [anos] } & \multirow{3}{*}{ Total } & \multirow{3}{*}{$p$} \\
\hline & & & \multicolumn{2}{|c|}{7} & \multicolumn{2}{|c|}{8} & \multicolumn{2}{|c|}{9} & \multicolumn{2}{|c|}{10} & & \\
\hline & & & $\mathrm{n}$ & $\%$ & $\mathrm{n}$ & $\%$ & $\mathrm{~N}$ & $\%$ & $n$ & $\%$ & & \\
\hline \multirow{6}{*}{$\begin{array}{l}\text { Estágio } \\
\text { Inicial }\end{array}$} & \multirow{3}{*}{$\begin{array}{l}\text { Agachamento prepa- } \\
\text { ratório inconsistente }\end{array}$} & Ausência & $8^{\mathrm{a}}$ & 5,8 & $20^{\mathrm{b}}$ & 14,6 & 19 & 13,9 & 21 & 15,3 & 68 & \\
\hline & & Presença & 2 & 1,5 & 9 & 6,6 & $35^{f}$ & 25,5 & 23 & 16,8 & 69 & \multirow[b]{2}{*}{$0,019^{*}$} \\
\hline & & Total & 10 & 7,3 & 29 & 21,2 & 54 & 39,4 & 44 & 32,1 & 137 & \\
\hline & \multirow{3}{*}{$\begin{array}{l}\text { Braços não coordena- } \\
\text { dos com o tronco e a } \\
\text { ação da perna }\end{array}$} & Ausência & $10^{c}$ & 7,3 & $23^{d}$ & 16,8 & 27 & 19,7 & 23 & 16,8 & 83 & \multirow{3}{*}{$0,001^{*}$} \\
\hline & & Presença & 0 & 0 & 6 & 4,4 & $27^{9}$ & 19,7 & 21 & 15,3 & 54 & \\
\hline & & Total & 10 & 7,3 & 29 & 21,2 & 54 & 39,4 & 44 & 32,1 & 137 & \\
\hline \multirow{3}{*}{$\begin{array}{l}\text { Estágio } \\
\text { Maduro }\end{array}$} & \multirow{3}{*}{$\begin{array}{l}\text { Elevação dos braços } \\
\text { coordenada e simul- } \\
\text { tânea }\end{array}$} & Ausência & 4 & 2,9 & 21 & 15,3 & $49^{e}$ & 35,8 & 39 & 28,5 & 113 & \multirow{3}{*}{$0,0001^{*}$} \\
\hline & & Presença & $6^{\mathrm{h}}$ & 4,4 & 8 & 5,8 & 5 & 3,6 & 5 & 3,6 & 24 & \\
\hline & & Total & 10 & 7,3 & 29 & 21,2 & 54 & 39,4 & 44 & 32,1 & 137 & \\
\hline
\end{tabular}

Nota: * Associação estatisticamente significativa entre as faixas etárias e 0 elemento do estágio de desenvolvimento

Ausência: (a) entre agachamento inconsistente e 7 anos $(R A=2,0)$; (b) entre agachamento inconsistente e 8 anos $(R A=2,3)$; (c) entre braços não coordenados e 7 anos ( $R A=2,6)$; (d) entre braços não coordenados e 8 anos; (e) entre elevação dos braços e 9 anos (RA=2,3);

Presença: (f) entre agachamento inconsistente e 9 anos ( $R A=2,7) ;(g)$ entre braços não coordenados e 9 anos (RA=2,0); (h) entre elevação dos braços e 7 $\operatorname{anos}(\mathrm{RA}=3,7)$. 
$\mathrm{Na}$ Tabela 2, considerando o maior valor do resíduo ajustado do teste qui-quadrado, no estágio inicial de desenvolvimento, observa-se que o agachamento preparatório inconsistente está associado a idade de nove anos, enquanto que a sua ausência está associada as idades de sete e oito anos. A ausência de braços não coordenados com o tronco e a ação da perna está associada com as idades de sete e oito anos, enquanto a sua presença está associada com crianças de nove anos.

Em relação ao aspecto da sequência desenvolvimentista no Estágio Maduro, observa-se que a presença de elevação dos braços coordenada e simultânea está associada com crianças na idade de sete anos e a ausência em crianças na aos nove anos de idade.

As tabelas 3 a 5 apresentam, respectivamente, as associações significativas entre os elementos da sequência desenvolvimentista - estágio inicial, elementar, maduro e a faixa de altura alcançada.

Tabela 3. Associação entre elementos do estágio inicial da sequência desenvolvimentista do salto vertical e a faixa de altura alcançada pelas crianças.

\begin{tabular}{|c|c|c|c|c|c|c|c|c|}
\hline \multirow{3}{*}{$\begin{array}{l}\text { Sequência desenvolvimentista } \\
\text { Estágio Inicial }\end{array}$} & & \multicolumn{4}{|c|}{ Faixa de Altura Alcançada [cm] } & & & \multirow{3}{*}{$p$} \\
\hline & & \multicolumn{2}{|c|}{$0-20$} & \multicolumn{2}{|c|}{$20-40$} & \multicolumn{2}{|c|}{ Total } & \\
\hline & & $\mathrm{n}$ & $\%$ & $\mathrm{~N}$ & $\%$ & $\mathrm{n}$ & $\%$ & \\
\hline \multirow{3}{*}{$\begin{array}{l}\text { Agachamento preparatório } \\
\text { inconsistente }\end{array}$} & Ausência & 17 & 12,4 & $51^{\mathrm{a}}$ & 37,2 & 68 & 49,6 & \multirow{3}{*}{$0,0001^{*}$} \\
\hline & Presença & $44^{e}$ & 32,1 & 25 & 18,2 & 69 & 50,4 & \\
\hline & Total & 61 & 44,5 & 76 & 55,5 & 137 & 100,0 & \\
\hline \multirow{3}{*}{$\begin{array}{l}\text { Extensão insuficiente do corpo ao } \\
\text { impulsionar }\end{array}$} & Ausência & 24 & 17,5 & $51^{b}$ & 37,2 & 75 & 54,7 & \multirow{3}{*}{$0,001^{*}$} \\
\hline & Presença & $37^{f}$ & 27,0 & 25 & 18,2 & 62 & 45,3 & \\
\hline & Total & 61 & 44,5 & 76 & 55,5 & 137 & 100,0 & \\
\hline \multirow{3}{*}{$\begin{array}{l}\text { Braços não coordenados com o } \\
\text { tronco e a ação da perna }\end{array}$} & Ausência & 30 & 21,9 & $53^{c}$ & 38,7 & 83 & 60,6 & \multirow{3}{*}{$0,014^{*}$} \\
\hline & Presença & $31^{g}$ & 22,6 & 23 & 16,8 & 54 & 39,4 & \\
\hline & Total & 61 & 44,5 & 76 & 55,5 & 137 & 100,0 & \\
\hline \multirow{3}{*}{ Baixa altura alcançada } & Ausência & 12 & 8,8 & $67^{d}$ & 48,9 & 79 & 57,7 & \multirow{3}{*}{$0,0001^{*}$} \\
\hline & Presença & $49^{h}$ & 35,8 & 9 & 6,6 & 58 & 42,3 & \\
\hline & Total & 61 & 44,5 & 76 & 55,5 & 137 & 100,0 & \\
\hline
\end{tabular}

Nota: * Associação estatisticamente significativa entre as faixas de altura de salto vertical e os elementos dos estágios de desenvolvimento Ausência: (a) entre agachamento inconsistente e $20-40 \mathrm{~cm}(\mathrm{RA}=4,6)$; (b) entre extensão insuficiente do corpo e $20-40 \mathrm{~cm}(\mathrm{RA}=3,2)$; (c) entre braços não coordenados e 20-40 cm (RA=2,4); (d) entre baixa altura alcançada e 20-40 cm ( $R A=8,1)$;

Presença: (e) entre agachamento inconsistente e $0-20 \mathrm{~cm}(R A=4,6)$; ( $(\mathrm{f})$ entre extensão insuficiente do corpo e $0-20 \mathrm{~cm}(\mathrm{Ra}=3,2) ;(\mathrm{g})$ entre braços não coordenados e 0-20 cm (RA=2,4); (h) entre baixa altura alcançada e 0-20 cm (RA=8,1).

Na tabela 3, a presença de agachamento preparatório inconsistente, a extensão insuficiente do corpo ao impulsionar e os braços não coordenados com o tronco e a ação da perna estão associados às menores alturas de salto. A presença do elemento baixa altura alcançada apresentou-se mais frequente na faixa de altura $0-20 \mathrm{~cm}$, o que reforça a qualidade da análise realizada em relação à configuração total do corpo.

$\mathrm{Na}$ tabela 5, a presença de extensão firme dos quadris, joelhos e tornozelos, a elevação dos braços coordenada e simultânea e a elevação do braço de alcance com inclinação do ombro combinada com abaixamento 
do outro braço, no auge do voo, estão associadas com as maiores alturas de salto. A presença de inclinação da cabeça para cima, com olhos focalizados no alvo, apresentou associação com as menores alturas de salto.

Tabela 4. Associação entre elementos do estágio elementar da sequência desenvolvimentista do salto vertical e a faixa de altura alcançada pelas crianças.

\begin{tabular}{|c|c|c|c|c|c|c|c|c|}
\hline \multirow{3}{*}{$\begin{array}{l}\text { Sequência desenvolvimentista } \\
\text { Estágio Elementar }\end{array}$} & & \multicolumn{4}{|c|}{ Faixa de Altura Alcançada[cm] } & \multirow{2}{*}{\multicolumn{2}{|c|}{ Total }} & \multirow{3}{*}{$p$} \\
\hline & & \multicolumn{2}{|c|}{$0-20$} & \multicolumn{2}{|c|}{$20-40$} & & & \\
\hline & & $n$ & $\%$ & $n$ & $\%$ & $n$ & $\%$ & \\
\hline \multirow{3}{*}{$\begin{array}{l}\text { Inclinação para frente exagerada durante o } \\
\text { agachamento }\end{array}$} & Ausência & $60^{a}$ & 43,8 & 65 & 47,4 & 125 & 91,2 & \multirow{3}{*}{$0,008^{*}$} \\
\hline & Presença & 1 & 0,7 & $11^{c}$ & 8,0 & 12 & 8,8 & \\
\hline & Total & 61 & 44,5 & 76 & 55,5 & 137 & 100,0 & \\
\hline \multirow{3}{*}{$\begin{array}{l}\text { Braços tentam auxiliar o vôo e o equilíbrio, } \\
\text { mas em geral não igualmente. }\end{array}$} & Ausência & $15^{b}$ & 10,9 & 9 & 6,6 & 24 & 17,5 & \multirow{3}{*}{$0,051^{*}$} \\
\hline & Presença & 46 & 33,6 & $67^{d}$ & 48,9 & 113 & 82,5 & \\
\hline & Total & 61 & 44,5 & 76 & 55,5 & 137 & 100,0 & \\
\hline
\end{tabular}

Nota: * Associação estatisticamente significativa entre as faixas de altura de salto vertical e os elementos dos estágios de desenvolvimento Ausência: (a) entre inclinação para frente e $0-20 \mathrm{~cm}(R A=2,6)$; (b) entre braços tentam auxiliar o vôo e $0-20 \mathrm{~cm}(R A=2,0)$.

Presença: (c) entre inclinação para frente e $20-40 \mathrm{~cm}(\mathrm{RA}=2,6)$; (d) entre braços tentam auxiliar o vôo e $20-40 \mathrm{~cm}(\mathrm{RA}=2,0)$.

Na tabela 4, a inclinação para frente exagerada durante 0 agachamento e os braços tentando auxiliar o vôo e o equilíbrio, mas em geral, não igualmente, estão associadas às maiores alturas de salto.

Tabela 5. Associação entre elementos do estágio maduro da sequência desenvolvimentista do salto vertical e a faixa de altura alcançada pelas crianças.

\begin{tabular}{|c|c|c|c|c|c|c|c|c|}
\hline \multirow{3}{*}{$\begin{array}{l}\text { Sequência desenvolvimentista } \\
\text { Estágio Maduro }\end{array}$} & & \multicolumn{4}{|c|}{ Faixa de Altura Alcançada [cm] } & & & \multirow{3}{*}{$p$} \\
\hline & & \multicolumn{2}{|c|}{$0-20$} & \multicolumn{2}{|c|}{$20-40$} & \multicolumn{2}{|c|}{ Total } & \\
\hline & & $\mathrm{n}$ & $\%$ & $\mathrm{n}$ & $\%$ & $\mathrm{n}$ & $\%$ & \\
\hline \multirow{3}{*}{$\begin{array}{l}\text { Extensão firme dos quadris, joelhos e } \\
\text { tornozelos }\end{array}$} & Ausência & $42^{\mathrm{a}}$ & 30,7 & 38 & 27,7 & 80 & 58,4 & \multirow{3}{*}{$0,026^{*}$} \\
\hline & Presença & 19 & 13,9 & $38^{e}$ & 27,7 & 57 & 41,6 & \\
\hline & Total & 61 & 44,5 & 76 & 55,5 & 137 & 100,0 & \\
\hline \multirow{3}{*}{$\begin{array}{l}\text { Elevação dos braços coordenada e simultâ- } \\
\text { nea }\end{array}$} & Ausência & $57^{\mathrm{b}}$ & 41,6 & 56 & 40,9 & 113 & 82,5 & \multirow{3}{*}{$0,002^{*}$} \\
\hline & Presença & 4 & 2,9 & $20^{f}$ & 14,6 & 24 & 17,5 & \\
\hline & Total & 61 & 44,5 & 76 & 14,6 & 137 & 100,0 & \\
\hline \multirow{3}{*}{$\begin{array}{l}\text { Inclinação da cabeça para cima com olhos } \\
\text { focalizados no alvo }\end{array}$} & Ausência & 15 & 10,9 & $38^{\mathrm{c}}$ & 27,7 & 53 & 38,7 & \multirow{3}{*}{$0,002^{*}$} \\
\hline & Presença & $46^{9}$ & 33,6 & 38 & 27,7 & 84 & 61,3 & \\
\hline & Total & 61 & 44,5 & 76 & 55,5 & 137 & 100,0 & \\
\hline \multirow{3}{*}{$\begin{array}{l}\text { Elevação do braço de alcance com inclina- } \\
\text { ção do ombro combinada com abaixamen- } \\
\text { to do outro braço no auge do voo }\end{array}$} & Ausência & $57^{d}$ & 41,6 & 50 & 36,5 & 107 & 78,1 & \multirow{3}{*}{$0,0001^{*}$} \\
\hline & Presença & 4 & 2,9 & $26^{h}$ & 19,0 & 30 & 21,9 & \\
\hline & Total & 61 & 44,5 & 76 & 55,5 & 137 & 100,0 & \\
\hline
\end{tabular}

Nota: * Associação estatisticamente significativa entre as faixas de altura de salto vertical e os elementos dos estágios de desenvolvimento Ausência: (a) entre extensão firme dos quadris e 0-20 cm (RA=2,2); (b) entre elevação dos braços e $0-20 \mathrm{~cm}(R A=3,0)$; (c) entre inclinação da cabeça e 20$40 \mathrm{~cm}(\mathrm{RA}=3,0)$; (d) entre elevação do braço de alcance e $0-20 \mathrm{~cm}(\mathrm{RA}=3,9)$;

Presença: (e) entre extensão firme dos quadris e 20-40 cm (RA=2,2); (f) entre elevação dos braços e 20-40 cm (RA=3,0); (g) entre inclinação da cabeça e $0-20 \mathrm{~cm}(\mathrm{RA}=3,0) ;(\mathrm{h})$ entre elevação do braço de alcance e $20-40 \mathrm{~cm}(\mathrm{RA}=3,9)$.

\section{DISCUSSÃO}

Para este estudo, as discussões consideram os resultados em relação à sequência desenvolvimentista do salto vertical e também os resultados da faixa etária e do desempenho de salto. Entende-se que vale ressaltar a limi- 
tação deste estudo em não investigar o histórico de experiências motoras das crianças e também, por não verificar a associação entre a faixa etária e a altura do salto. Também há de se considerar as características inerentes à avaliação qualitativa, em que os elementos da sequência desenvolvimentista são analisados a partir da interpretação visual do avaliador ${ }^{18}$. Na estimativa da altura do salto, entende-se que a flexão do joelho na fase aérea antes da aterrissagem poderia aumentar o tempo de vôo. Em se tratando de populações de crianças, considera-se tal estratégia improvável de ter acontecido, pois a amostra não tinha conhecimento sobre os procedimentos de cálculo; entretanto, essa pode ser uma limitação de estudo.

Ao analisar os elementos do estágio de desenvolvimento motor, observamos algumas associações significativas com as faixas etárias. Para crianças de sete anos, constataram-se a ausência de agachamento preparatório inconsistente e de braços não coordenados com o tronco e a ação da perna e a presença de elevação de braços coordenados e simultânea. Para as crianças de oito anos, foram constatados a ausência de agachamento preparatório inconsistente e de braços não coordenados com o tronco e a ação da perna. A presença de agachamento preparatório inconsistente e a ação de braços não coordenados com o tronco e a perna e a ausência de elevação dos braços, coordenada e simultânea, aconteceram prioritariamente nas crianças de nove anos de idade. Não foram encontradas associações locais significativas entre os elementos do desenvolvimento motor do salto e a idade de 10 anos.

A diversidade de aspectos relacionados às faixas etárias possivelmente pode ser ponderada pelos achados de Labiadh et al. ${ }^{19}$ que, ao avaliar crianças entre 3,5 e 7,5 anos, observaram que estas não apresentavam necessariamente a mesma coordenação nos modos de salto.

Entende-se que para atingir o estágio maduro do padrão motor saltar, as experiências motoras anteriores são fundamentais ${ }^{1}$. Há de se considerar a limitação do presente estudo em não controlar as experiências das crianças estudadas, o que pode ter afetado o resultado em relação ao nível de desenvolvimento motor das crianças.

Em relação ao desempenho, foi possível diagnosticar associações com os elementos do estágio de desenvolvimento motor. Para esta análise, foram considerados desempenhos abaixo e acima de $20 \mathrm{~cm}$, altura escolhida por representar a mediana desta variável na distribuição da amostra.

O desempenho de salto abaixo de $20 \mathrm{~cm}$ esteve associado à presença do agachamento preparatório inconsistente; da extensão insuficiente do corpo ao impulsionar; da ação dos braços de forma não coordenada com o tronco e as pernas e da inclinação da cabeça para cima com olhos focalizados no alvo.

O desempenho de salto acima de $20 \mathrm{~cm}$ esteve associado à presença de inclinação para frente exagerada durante o agachamento; da ação dos braços tentando auxiliar o vôo e o equilíbrio, mas em geral não igualmente; de extensão firme dos quadris, joelhos e tornozelos; da elevação dos braços coordenada e simultânea e da elevação do braço de alcance com inclinação do ombro combinada com abaixamento do outro braço no auge do vôo. 
Nos aspetos listados acima, observa-se uma prevalência de fatores relacionados à ação dos membros superiores em relação aos inferiores. Inicialmente, pode parecer que os achados neste trabalho sejam conflitantes com a literatura, pois existe indicação que, para saltos submáximos, uma estratégia consistente refere-se à contribuição máxima das articulações distais e mínima das articulações proximais ${ }^{20}$.

No entanto, este comportamento pode ser explicado pelas informações de $\mathrm{Hara}^{21}$, onde afirma que o incremento da altura do salto resulta do aumento do trabalho dos membros inferiores, que é considerada como tendo sido provocada pela carga adicional na extremidade mais baixa devido ao balanço do braço. Corroborando esta análise, Mourão e Gonçalves ${ }^{22}$ dizem que o balanço dos membros superiores na realização de um salto vertical leva a um aumento do desempenho.

Parece-nos que a contribuição das informações observadas neste trabalho vão ao encontro da ênfase no desenvolvimento dos segmentos corporais para o desempenho da altura do salto. Tanto para a análise da associação dos elementos dos estágios da sequência do desenvolvimento motor com a idade quanto com o desempenho do salto, destacamos a contribuição dos membros superiores.

Assim, uma vez evidenciado que, nas associações significativas entre o padrão motor e o desempenho, há um predomínio da contribuição dos membros superiores, parece-nos importante que no processo de ensino-aprendizagem desta habilidade motora seja dado ênfase aos movimentos dos braços de forma coordenada e simultânea. Tal característica é ressaltada na literatura para um padrão maduro ${ }^{1}$.

\section{CONCLUSÕES}

Analisando os elementos desenvolvimentistas da configuração total do corpo humano na habilidade do salto vertical em escolares, este estudo permite supor a existência de associações significativas com a faixa etária e o desempenho.

Em relação à faixa etária, observa-se uma inversão nos níveis de padrões, pois as crianças mais novas apresentaram características de padrões maduros. Quanto ao desempenho, houve evidência da contribuição dos membros superiores para a realização do salto.

Considerando as limitações do estudo, sugere-se que, para futuras pesquisas, as experiências motoras sejam investigadas, visando uma análise desenvolvimentista com maior profundidade.

\section{REFERÊNCIAS BIBLIOGRÁFICAS}

1. Gallahue DL, Ozmun JC. Compreendendo o desenvolvimento motor: bebês, crianças, adolescentes e adultos. São Paulo: Phorte Editora; 2001.

2. Fagenbaum MS, Warren GD. Jump landing strategies in male and female college athletes and the implications of such strategies for anterior cruciate ligament injury. Am J Sports Med 2003;31(2):233-40. 
3. Powell JW, Barber-Foss KD. Injury patterns in selected high school sports: A review of the 1995-1997 seasons. J Athl Train 1999;34(3):277-84.

4. Haguenauer M, Legreneurm P, Monteil KM. Vertical jumping reorganization with aging: a kinematic comparison between young and elderly men. J Appl Biomech 2005; 21(3):236-46.

5. Okely AD, Booth ML, Chey T. Relationships between body composition and fundamental movement skills among children and adolescents. Res Q Exerc Sport 2004;75(3):238-47.

6. Eisenmann JC, Malina R. M. Age- and sex-associated variation in neuromuscular capacities of adolescent distance runners. J Sports Sci 2003;21(7):551-7.

7. Çoksevim B, Çaksen H. Evaluation of exercise performance in healthy turkish adolescents. Int J Neurosci 2005;115(7):1033-1039.

8. Carvalhal M, Vasconcelos-Raposo J. Diferenças entre géneros nas habilidades: correr, saltar, lançar e pontapear. Motricidade 2007;3(3):44-56.

9. Okely AD, Booth ML. Mastery of fundamental movement skills among children in New South Wales: prevalence and sociodemographic distribution. J Sci Med Sport 2004;7(3):358-72.

10. Harrison AJ, Ryan W, Hayes K. Functional data analysis of joint coordination in the development of vertical jump performance. Sports Biomech 2007;6(2):199-214.

11. McKay H, Tsang G, Heinonen A, MacKelvie K, Sanderson D, Khan KM. Ground reaction forces associated with an effective elementary school based jumping intervention. Br J Sports Med 2005;39(1):10-4.

12. Wikstrom EA, Tillman MD, Kline KJ, Borsa PA. Gender and limb differences in dynamic postural stability during landing. Clin J Sport Med 2006;16(4):311-5.

13. Oxyzoglou N, Kanioglou A, Rizos S, Mavridis G, Kabitsis C. Muscular strength and jumping performance after handball training versus physical education program for pre-adolescent children. Percept Mot Skills 2007;104:1282-8.

14. Gallahue DL, Donnelly FC. Educação física desenvolvimentista para todas as crianças. 4. ed. São Paulo: Phorte; 2007.

15. Barros MVG, Reis RSR, Hallal PR, Florindo AA. Análise de dados em saúde. 2.ed. Recife: Edupe; 2005.

16. Callegari-Jacques SM. Bioestatística: princípios e aplicações. Porto Alegre: Artmed; 2003.

17. Goldacre MJ, Turner G, Lambert TW. Variation by medical school in career choices of UK graduates of 1999 and 2000. Med Educ 2004;38:249-58.

18. Gallahue DL. A classificação das habilidades de movimento: um caso para modelos multidimensionais. Rev Educ Fís/UEM 2002; 13(2):105-111.

19. Labiadh L, Ramanantsoa MM, Golomer E. Preschool-aged children's jumps: imitation performances. J Electromyogr Kinesiol 2010; 20(2):322-9.

20. Vanrentterghem J, Lees A, Lenoir M, Aerts P, De Clerq D . Performing the vertical jump: movement adaptations for submaximal jumping. Hum Mov Sci 2004;22:713727.

21. Hara M, Shibayama A, Takeshita D, Fukashiro S. The effect of arm swing on lower extremities in vertical jumping. J Biomech 2006;39(13):2503-11.

22. Mourão PJM, Gonçalves FJM. A utilização dos membros superiores nos saltos verticais: estudo comparativo entre um salto sem contramovimento sem a utilização dos membros superiores e um salto sem contramovimento com a utilização dos membros superiores. Motriz 2008;4(4):23-8. 